\title{
Uncertain multi objective multi item four dimensional transportation problem with vehicle speed
}

\author{
A.N.Revathi ${ }^{1 *}$, S.Mohanaselvi ${ }^{1}$, Dipak Kumar Jana ${ }^{2}$ \\ \{revathin1@ srmist.edu.in ${ }^{1}, \underline{\text { mohanass@ }}$ srmist.edu.in $^{2}$, dipakjana@gmail.com ${ }^{3}$ \}
}

\begin{abstract}
Department of Mathematics, Faculty of Engineering and Technology, SRM Institute of Science and Technology, Kattankulathur, Chennai - 603 203, Tamil Nadu, India ${ }^{1, *}$, School of Applied Sciences \& Humanities, Haldia Institute of Technology, Haldia, Purba MidnaPur-721657, West Bengal, India. ${ }^{2}$
\end{abstract}

\begin{abstract}
The In this work, multi objectives multi item four dimensional transportation problem with vehicle speed consisting of uncertain variable parameters is studied. Due to bad road conditions, the rate of variation of speed of the vehicle may vary in different routes and the speed of different vehicles are also considered first time in the time minimization transportation model. Equivalent deterministic models for the uncertain programming model are proposed by using chance constrained and expected value method. Finally goal programming technique is applied to derive a suitable compromise solution of the uncertain multi objective multi item four dimensional transportations problems with consideration of vehicle speed. An illustrative real life situation is discussed and optimal results are displayed to demonstrate the proposed methodology..
\end{abstract}

Keywords: Four dimensional transportation problem _ Uncertain variables _ Goal programming.

\section{Introduction}

The primitive transportation problematic presented by Hitchcock[1] in 1941 and it is developed by Koopmans[2]. Currently transportation problem(TP) with single objective are not sufficient in today's market to manage day-to-day decision making problem. Thus, in order to maximize the profit for the firm, we consider many objectives at a time. Since there is no connection between the cost parameter of various objectives of the Transportation Problem: these are considered as conflicting and commensurable model of the multi objective transportation problem [MOTP]. Well known researchers like Lee et al 1973[3], Pandian et al 2011[4], Quddoos et al 2013[5] and Nomani et al 2017[6] etc., therefore worked on more efficient process to solve MOTP.

The transportation problem with conveyance is termed as solid transportation problem [STP] and it was presented by Haley[7]. Numerous models like multi objective solid transportation problem, solid transportation with secure responsibility etc., have been developed under STP from then. In global competition, STP is a major factor as it minimize solid transportation cost, time taken to provide the service and distance. Applying zero point principle Pandian et al[8] have provided a solution methodology for obtaining the minimized transported cost of STP. Furthermore, he extended his work by providing cost sensitivity of ranges[9]. 
When we consider real life problem the presence of multiple routes/roads bring out other factor such as the condition of roads, the different distances between the routes. However all the transportation modes and routes may not be ideal. So while trying to maximize the profit, we need to factor in breakability. Also the breakability factor depends upon several other choices such as the roads condition, distance and the vehicle used. So considering the fair choice of routes plays a major role. A four dimensional solid transportation problem [4DSTP] is a STP wherein along with the conveyances different routes and vehicles are considered. The distinguishing factor between TP's, STP's and 4DTP's is that the distance too is considered. To solve the time minimization transportation problem, the speed of the vehicle also play a vital role. It might be possible that shortest road routes might not be in best condition. In such routes might affect the speed of transportation. The rate of disturbances of speed has not been considered by any researchers till date. Considering multiple objective and multiple product in a 4DTP converts a 4DTP into four dimensional multi objective multi item transportation problem [4DMOMITP].

real life situation all the parameters required for the STP are not always accurate or available. Contradicting in the STP problem we considered, we assumed to be fairly accurate. The parameters like availability of demand and constituent transportation charge are not exact continuously due to the uncertainty in human judgment and lack of information which are uncontrollable factors. But these uncertainties can be considered by fuzzy sets given by Zadeh[10]. Multiple researchers who proposed methods to solve TP'sutilized fuzzy numbers for representing the parameters' uncertainty.

To solve MOTP, Zimmerman[11] gave fuzzy programming method. It was later studied by bit et al[12] to solve multi objective solid transportation problem [MOSTP] where in genetic algorithm was applied by Liu et al[3] where objective function coefficient were taken as fuzzy numbers. The Method of solving fuzzy MOSTP, taking all parameters as fuzzy numbers excluding decision variable was proposed by Ojha et al[13] to solve fully fuzzy MOTP, the Mehar's method was introduced by Gupta et al[14]. Deepika rani et al[15] presented a technique to obtain the optimal compromise solution of FFMOMISTP. In fuzzy environment (intuitionistic L-R type) and vehicle speed, Dipakkumar et al formulated MOMITP in four dimensions[16].

Although Zadeh's Fuzzy set theory[10] is widely applied in many uncertain models, it could not handle human uncertainty in some contexts involving incomplete information. As an attempt to deal with such indeterminacies, Liu founded uncertainty theory[17],[18]. Nowadays, doubt theory is considered as a mathematical division for modelling belief degrees and has been adopted in many mathematical models like uncertain programming, logic, graph, statistics and finance[19],[20],[21]. The belief degree of an indeterminate event to happen is measured by indeterminate amount. To simultaneously contract with uncertainty and randomness, the usage of random indeterminate variable and chance measure was also introduced by Liu[22]. Post that, he also presented uncertain random programming to model optimization problems containing more than one random variable. Gao[23], in his paper, newly proposed certain properties based on continuously uncertain measures. SeyyedMojtabaChasence[24] introduced uncertain linear fractional programming problem and also presented few methods to convert uncertain optimization problem as an equivalent deterministic problem. 
Liu[25] introduced a new uncertain multi objective programming and goal programming technique as a compromised method to solve uncertain multi objective programming using inverse uncertainty distribution. Uncertain goal programming method was proposed by Liu Chen[26] for an uncertain multi objective programming problem. To get the answer of the multi level indeterminate programming problems, Yao and Liu [27] developed programming methods. For an uncertain multi objective problem, a compromise programming models and interactive satisfied methods were presented by Zhou et al and Zhong et al[28],[29]. For uncertain STP, Cui and Sheng[30] presented an expected constrained model. Later, a transportation problem was studied by Guo et al[31] consisting of random supplies and uncertain costs. By using type-2 uncertain optimization methods, Yang et al[32] found the compromise solution for fixed charge STP. Multi objective multi item STP under uncertain situation was formulated by Hasan Dalman[33]. A solid transportation problem involving creation amalgamation was studied by Yuan Gao and SamarjitKar[34]. Motivated by above authors, we have tried to propose a solution methodology for an uncertain multi objective multi item four dimensional transportation model with vehicle speed[UMOMI4DTPS]. The prime motive of this prototypical is to exploit the income and minimize the transportation charge. In the time minimization objective function, the speed of the various conveyance and degradation of speed owing to poor road conditions have been factored in for the first time ever.

We created three different models. Out of the three models that we are presenting, First model has been procured considering the items whose breakability that depends on both conveyances and type of routes taken. The next one considers shipping of items that are damageable only because of its type. The last one involves shipping of non-breakable item. The equivalent classical models for UMOMI4DTPS are obtained by by predictable chance and value restraint technique. The models are demonstrated by numerical instance to know the efficiency of the suggested model for obtaining the best compromise solution.

The article has been structured thusly. We have presented the theories and descriptions of doubt theory which are used models in section 2. Notations used in the article is assumed in segment 3. In segment 4, the mathematical model of uncertain multi objective is introduced. Equivalent deterministic models by using expected value and chance constraint methodsare given in the sections 5 and 6 respectively. Fundamental ideas about goal programming technique and the procedure for solving the UMOMI4DTPS are given in section 7 and 8 respectively. A numerical example has been given in section 9. The discussion, practical implication, comparison and conclusions have been given in sections 10,11,12 and 13 respectively.

\section{Preliminaries}

Some fundamental concept of indecision theory, which have been use in the subsequent discussions are introduced below.

Definition 1: [20],[17] Let $\mathcal{L}$ be a $\sigma$-algebra of collection of events $\Lambda$ of a universal set $\Gamma$. A set function $\mathcal{M}$ is said to be indeterminate portion defined on the $\sigma$-algebra where 
$\mathcal{M}\{\Lambda\}$ designate the confidence grade with which we trust that the occasion will happens and satisfies the subsequent maxims:

1. Normality Axiom: For the universal set $\Gamma$, we have $\mathcal{M}\{\Gamma\}=1$.

2. Duality Axiom: For any event $\Lambda$, we have $\mathcal{M}\{\Lambda\}+\mathcal{M}\left\{\Lambda^{\mathrm{C}}\right\}=1$.

3. Subadditivity Axiom: For every countable sequence of events $\Lambda_{1}, \Lambda_{2}, \ldots$, we have $\mathcal{M}\left\{\bigcup_{i=1}^{\infty} \Lambda_{i}\right\} \leq \sum_{i=1}^{\infty} \mathcal{M}\left\{\Lambda_{i}\right\}$

4. Creation Axiom: Let $\left(\Gamma_{i}, \mathcal{L}_{i}, \mathcal{M}_{i}\right)$ be indecision spaces for $\mathrm{i}=1,2,3, \ldots$. The produce uncertain quantity is an uncertain measure holds

$$
\mathcal{M}\left\{\prod_{i=1}^{\infty} \wedge_{i}\right\}=\bigwedge_{i=1}^{\infty} \mathcal{M}\left\{\wedge_{i}\right\} \text { where } \Lambda_{i} \in \mathcal{L}_{i} \text { for } i=1,2,3, \ldots ., \infty \text {. }
$$

Definition 2: [17] A function $\xi:(\Gamma, \mathcal{L}, \mathcal{M}) \rightarrow \Re$ is said to be an uncertain variable such that $\{\xi \in \mathrm{B}\}=\{\gamma \in \Gamma / \xi(\gamma) \in \mathrm{B}\}$ is an event for any Borel set $B$ of real numbers.

Definition 3: [17] An indeterminate variable $\xi$ defined on the uncertainty space $(\Gamma, \mathcal{L}, \mathcal{M})_{\text {is said to be non- negative if }} \mathcal{M}\{\xi<0\}=0$ and positive if $\mathcal{M}\{\xi \leq 0\}=0$.

Definition 4: [17] For any real number ${ }^{x}$, the indecision distribution ${ }^{\phi(x)}$ of an uncertain variable $\xi$ is defined by $\phi(x)=\mathcal{M}\{\xi \leq x\}$.

Definition 5: Let $\phi(x)$ be the regular indecision distribution of an uncertain variable $\xi$. Then $\phi^{-1}(\alpha)$ is called inverse uncertainty distribution of $\xi$ and it exists on $(0,1)$.

Definition 6: [17] The uncertain variable $\xi_{i} \quad(i=1,2, \ldots, n)$ are said to be autonomous if

$$
\mathcal{M}\left\{\bigcap_{i=1}^{n}\left(\xi_{i} \in B_{i}\right)\right\}={ }_{i=1}^{n} \mathcal{M}\left(\xi_{i} \in B_{i}\right)
$$

where $^{B_{i}} \quad(i=1,2, \ldots n)$ are called Borel sets of real numbers.

Theorem 1: let $\xi$ be an uncertain variable with regular uncertain distribution function $\psi$.Then its $\alpha-$ optimistic value and $\alpha-$ pessimistic values are

$$
\xi_{\text {sup }}(\alpha)=\psi^{-1}(1-\alpha), \quad \xi_{\text {inf }}(\alpha)=\psi^{-1}(\alpha) .
$$

Theorem 2: [18] The regular uncertainty distributions of independent uncertain variables $\xi_{i} \quad(i=1,2,3, \ldots, m, \ldots, n) \quad$ are $\phi_{i} \quad(i=1,2, \ldots, m, \ldots, n) \quad$ respectively. If the function $f\left(x_{1}, x_{2}, \ldots, x_{m}, \ldots, x_{n}\right)$ is strictly increasing and strictly decreasing with respect to $x_{1}, x_{2}, \ldots, x_{m}$ and $x_{m+1}, x_{m+2}, \ldots, x_{n}$ respectively then the uncertain variable $\xi=f\left(\xi_{1}, \xi_{2}, \ldots \xi_{m}, \ldots, \xi_{n}\right)$ has an inverse uncertainty distribution $\psi^{-1}(\alpha)=f\left(\phi_{1}^{-1}(\alpha), \phi_{2}^{-1}(\alpha), \ldots, \phi_{m}^{-1}(\alpha), \phi_{m+1}^{-1}(1-\alpha), \phi_{m+2}^{-1}(1-\alpha), \ldots, \phi_{n}^{-1}(1-\alpha)\right)$

Definition 7: [17] The expected value of uncertain variable $\xi$ is given by 


$$
E(\xi)=\int_{0}^{\infty} \mathcal{M}\{\xi \geq x\} d x-\int_{-\infty}^{0} \mathcal{M}\{\xi \leq x\} d x
$$

This is valid only if at least one of the integral is finite.

Theorem 3: [35] The regular uncertainty distributions of independent uncertain variables $\xi_{i} \quad(i=1,2, \ldots, m, \ldots, n)$ are $\phi_{i} \quad(i=1,2, \ldots, m, \ldots, n)$ respectively. If the function $f\left(x_{1}, x_{2}, . ., x_{m} . ., x_{n}\right)$ is strictly increasing and strictly decreasing w.r.to $x_{1}, x_{2}, \ldots, x_{m}$ and $x_{m+1}, x_{m+2}, \ldots, x_{n}$ respectively, then

$$
E(\xi)=\int_{0}^{1} f\left(\phi_{1}^{-1}(\alpha), \ldots . \phi_{m}^{-1}(\alpha), \phi_{m+1}^{-1}(1-\alpha), \ldots \phi_{n}^{-1}(1-\alpha)\right) d \alpha
$$

From the above theorem, we know that

$$
E(\xi)=\int_{0}^{1} \phi^{-1}(\alpha) d \alpha
$$

where $\xi$ is an uncertain variable with regular uncertainty distribution $\Phi$.

Definition 8: [17] A linear uncertain variable $\xi$ is defined as

$$
\phi(x)= \begin{cases}0 & , \text { if } x \leq l \\ \frac{x-l}{m-l} & , \text { if } l \leq x \leq m \\ 1 & , \text { if } x \geq m\end{cases}
$$

denoted by $\mathrm{L}(\mathrm{l}, \mathrm{m})$, where in $1, \mathrm{~m} \in \mathrm{R}$ with $1<\mathrm{m}$.

The inverse delivery function of a linear uncertain variable $\mathrm{L}(1, \mathrm{~m})$ is given by

$$
\phi^{-1}(\alpha)=(1-\alpha) l+\alpha m
$$

Where $\alpha=0.5$ and its expected value is given by

$$
E[\xi]=\frac{l+m}{2}
$$

\section{NOMENCLATURE}

The following notations have been considered for constructing the proposed two models:

$\mathrm{m}$ number of origin

$\mathrm{n} \quad$ number of destination

$\mathrm{K}$ number of mode of transport

$\mathrm{R}$ number of transportation routes

$\mathrm{T}$ number of products

$\tilde{Z}^{w}$ uncertain objective functions, where $\mathrm{w}=1,2, \ldots$

$\tilde{P}_{i g} i^{\text {th }}$ - origin, ${ }^{\text {th }}$ good's purchasing price

$\tilde{S}_{j g} j^{\text {th }}$ destination, ${ }^{\text {th }}$ good's selling price 
$\tilde{C}_{i j k r g}$ the unit transportation cost of ${ }^{\text {th }}$ good from $i^{\text {th }} \quad$ origin to $j^{j^{\text {th }}}$ terminus by $k^{\text {th }}$ transport via $r^{\text {th }}$ road per unit distance.

$\beta_{i j k r g}$ the rate of breakability per unit distance of ${ }^{g \text { th }}$ good from $i^{t^{\text {th }}}$ origin to the $j^{\text {th }}$ terminus by $k^{\text {th }}$ transport via $r^{\text {th }}$ road.

$d_{i j r}$ distance from $i^{\text {th }}$ origin to $j^{\text {th }}$ terminus via $r^{\text {th }}$ road.

$V_{k}$ speed of the $k^{\text {th }}$ transport

$\gamma_{i j r}$ rate of disturbance of the speed due to $r^{\text {th }}$ road from $i^{\text {th }}$ origin to $j^{\text {th }}$ destination.

$\tilde{\delta}_{i j k g}$ unloadingand loading time of the ${ }^{g^{\text {th }}}$ good with admiration to the transportmovement from $i^{\text {th }}$ origin to $j^{\text {th }}$ destination by $k^{\text {th }}$ transport

$\tilde{a}_{i g}$ quantity of $g^{\text {th }}$ good available at $i^{\text {th }}$ origin

$\tilde{b}_{j g}$ therequirement of ${ }^{g^{t h}}$ good at the ${ }^{j^{\text {th }}}$ destination

$\tilde{e}_{k}$ capacity of a single vehicle of $k^{\text {th }}$ transport.

$D^{-}$negative deviational value.

$D^{+}$positive deviational value.

\section{Mathematical Formulation Of UMOMI4DTPS:}

A uncertain multi objective multi item four dimensional transportation problem with vehicle speed is formulated as follows. Let there be m origins $\mathrm{O}_{\mathrm{i}}(\mathrm{i}=1,2, \ldots \mathrm{m}), \mathrm{n}$ demands $D_{j}$ $(\mathrm{j}=1,2, \ldots \ldots \mathrm{n}), \mathrm{R}$ roads $Q_{r}(\mathrm{r}=1,2 \ldots . \mathrm{R}), \mathrm{G}$ goods ${ }^{\mathrm{P}_{g}}(\mathrm{~g}=1,2, \ldots \mathrm{G}), \mathrm{K}$ conveyances $E_{k}$ $(\mathrm{k}=1,2, \ldots . \mathrm{K})$. The objective of this transportation model is to maximize the profit and minimize the time taken for transportation. Estimating the exact amount of parameters that are related is not so simple in the present situation. Application of transportation problem in real life situation involves lot of uncertainties for the decision makers such as availability of raw materials, fluctuation of unit transportation cost and demands in destinations. Hence the following are some unmanageable factors in transportation problems.

1. The product availability at the origin may be uncertain, considering time factor.

2. The decision maker may not always know the unit transportation charge for the $1^{\text {st }}$ transportation operation.

3. The recently introduced products' total demand may be uncertain in the market.

So, we formulate considering all the parameters(availabilities, demands, purchase price, selling price, loading and unloading time, capacity of the conveyances and transportation cost) as uncertain variable which might be incorporated in the real life situation.

There are chances of goods or items are transported may be damaged during the transportation period. The reason for this breakability's are diverse. It may depend on the route through which the item is transported and also the nature of the goods that transported. 
Mainly the glass items, especially ceramics and china clay have this concerns. For some items damageability may be due to its type only. Some items may not be damageable at all. Considering the above factor, we have introduced three different models by involving the rate of breakability and are given as follows.

Model 1: In model 1, we consider the transporting goods that are vulnerable to breakability, that depend on different mode of transports along different routes. Here, we use $\beta_{i j k r g}$ the rate of breakability per unit distance from $i^{\text {th }}$ origin via $r^{\text {th }}$ road to $j^{\text {th }}$ destination for the $g^{\text {th }}$ goods via $k^{\text {th }}$ transport.

Model 2: This model considers the goods that are damageable because of their nature. $\beta_{g}$ is used to denote the rate of breakability per unit distance of the $g^{\text {th }}$ goods.

Model 3: This model considers non-breakable goods.

4.1. Model 1: UMOMI4DTPS with breakable items based on mode of transports, roads and goods

The maximizing the total profit is the first objective and minimizing the transportation time is the second objective of the model which are given below. The transporting time will be taken into consideration if the goods are transported from $i^{\text {th }}$ origin to $j^{\text {th }}$ terminus by $k^{\text {th }}$ transport via $r^{\text {th }}$ road.

$$
\left\{\begin{array}{l}
\operatorname{Max} \tilde{Z}_{1}=\sum_{i=1}^{m} \sum_{j=1}^{n} \sum_{k=1}^{K} \sum_{r=1}^{R} \sum_{g=1}^{G}\left[\tilde{S}_{j g}\left(1-\beta_{i j k r g} * d_{i j r}\right)-\tilde{P}_{i g}-\left(\tilde{c}_{i j k r g} * d_{i j r}\right)\right] * x_{i j k r g} \\
\operatorname{Min} \tilde{Z}_{2}=\sum_{i=1}^{m} \sum_{j=1}^{n} \sum_{k=1}^{K} \sum_{r=1}^{R} \frac{d_{i j r} * y_{i j k r}}{v_{k}\left(1-\gamma_{i j r}\right)}+\sum_{i=1}^{m} \sum_{j=1}^{n} \sum_{k=1}^{K} \sum_{r=1}^{R} \sum_{g=1}^{G} \tilde{\delta}_{i j k g} * x_{i j k r g} \\
\text { where, } y_{i j k r}=\left\{\begin{array}{l}
1, \text { if } \sum_{g=1}^{G} x_{i j k r g}>0, \\
0, \text { if } \sum_{g=1}^{G} x_{i j k r g}=0, \\
\text { subject to, } \\
\sum_{j=1}^{n} \sum_{k=1}^{K} \sum_{r=1}^{R} x_{i j k r g} \leq \tilde{a}_{i g}, i=1 \ldots m, g=1 \ldots G, \quad \text { (supply constraints) } \\
\sum_{i=1}^{m} \sum_{k=1}^{K} \sum_{r=1}^{R}\left(1-\left(\beta_{i j k r g} * d_{i j r}\right)\right) x_{i j k r g} \geq \tilde{b}_{j g}, j=1 \ldots n, g=1 \ldots G, \quad \text { (demand constraints) } \\
\sum_{i=1}^{m} \sum_{j=1}^{n} \sum_{r=1}^{R} \sum_{g=1}^{G} x_{i j k r g} \leq \tilde{e}_{k}, k=1 \ldots K, \quad(\text { Conveyance constraints) } \\
x_{i j k r g} \geq 0, \forall i, j, k, r, g .
\end{array}\right.
\end{array}\right.
$$

4.2 Model 2:UMOMI4DTPS with breakable items due to their nature

In this model we have formulated a case wherein the goods that are selected has damageability factor only because of its type. This model is same as of the previous model 
except the objective and constraint which depends upon $\beta_{i j k r t}$. So, the required model is developed as shown below:

$$
\left\{\begin{array}{l}
\operatorname{Max} \tilde{Z}_{1}=\sum_{i=1}^{m} \sum_{j=1}^{n} \sum_{k=1}^{K} \sum_{r=1}^{R} \sum_{g=1}^{G}\left[\tilde{S}_{j g}\left(1-\beta_{g} * d_{i j r}\right)-\tilde{P}_{i g}-\left(\tilde{c}_{i j k r g} * d_{i j r}\right)\right]^{*} x_{i j k r g} \\
\operatorname{Min} \tilde{Z}_{2}=\sum_{i=1}^{m} \sum_{j=1}^{n} \sum_{k=1}^{K} \sum_{r=1}^{R} \frac{d_{i j r} * y_{i j k r}}{v_{k}\left(1-\gamma_{i j r}\right)}+\sum_{i=1}^{m} \sum_{j=1}^{n} \sum_{k=1}^{K} \sum_{r=1}^{R} \sum_{g=1}^{G} \tilde{\delta}_{i j k g} * x_{i j k r g} \\
\text { where, } \quad y_{i j k r}=\left\{\begin{array}{l}
1, \text { if } \sum_{g=1}^{G} x_{i j k r g}>0, \\
0, \text { if } \sum_{g=1}^{G} x_{i j k r g}=0, \\
\text { subject to, } \\
\sum_{j=1}^{n} \sum_{k=1}^{K} \sum_{r=1}^{R} x_{i j k r g} \leq \tilde{a}_{i g}, i=1 \ldots m, g=1 \ldots G, \\
\sum_{i=1}^{m} \sum_{k=1}^{K} \sum_{r=1}^{R}\left(1-\left(\beta_{g} * d_{i j r}\right)\right) x_{i j k r g} \geq \tilde{b}_{j g} \forall j, g \\
\sum_{i=1}^{m} \sum_{j=1}^{n} \sum_{r=1}^{R} \sum_{g=1}^{G} x_{i j k r g} \leq \tilde{e}_{k}, k=1 \ldots K, \\
x_{i j k r g} \geq 0, \forall i, j, k, r, g .
\end{array}\right.
\end{array}\right.
$$

4.3.Model 3: UMOMI4DTPS with no damageable goods

In this model, we have formulated a problem which involves no breakable goods. As we cannot deal uncertain environment directly, the above problem with uncertain supplies, demands, costs, filling and receipt time and capacities could be converted as equivalent deterministic model by employing the expected value and chance constraint methods.

\section{Expected Value Models For UMOMI4DTPS}

In this section, we have introduced the equivalent deterministic models for UMOMI4DTPS using expected value method.

\subsection{Expected value method for Model 1}

Equivalent deterministic form for model 1 by using expected value method is given in (13).

Applying the properties of theories of expectation in the (13), we have (14). 


$$
\left\{\begin{array}{l}
\operatorname{Max} \tilde{Z}_{1}=\sum_{i=1}^{m} \sum_{j=1}^{n} \sum_{k=1}^{K} \sum_{r=1}^{R} \sum_{g=1}^{G}\left[\tilde{S}_{j g}-\tilde{P}_{i g}-\left(\tilde{c}_{i j k r g} * d_{i j r}\right)\right]^{*} x_{i j k r g} \\
\operatorname{Min} \tilde{Z}_{2}=\sum_{i=1}^{m} \sum_{j=1}^{n} \sum_{k=1}^{K} \sum_{r=1}^{R} \frac{d_{i j r} * y_{i j k r}}{v_{k}\left(1-\gamma_{i j r}\right)}+\sum_{i=1}^{m} \sum_{j=1}^{n} \sum_{k=1}^{K} \sum_{r=1}^{R} \sum_{g=1}^{G} \tilde{\delta}_{i j k g} * x_{i j k r g} \\
\text { where, } \quad y_{i j k r}=\left\{\begin{array}{l}
1, \text { if } \sum_{g=1}^{G} x_{i j k r g}>0, \\
0, \text { if } \sum_{g=1}^{G} x_{i j k r g}=0
\end{array}\right. \\
\text { subject to, } \\
\sum_{j=1}^{n} \sum_{k=1}^{K} \sum_{r=1}^{R} x_{i j k r g} \leq \tilde{a}_{i g}, i=1 \ldots m, g=1 \ldots G, \\
\sum_{i=1}^{m} \sum_{k=1}^{K} \sum_{r=1}^{R} x_{i j k r g} \geq \tilde{b}_{j g} \forall j, g \\
\sum_{i=1}^{m} \sum_{j=1}^{n} \sum_{r=1}^{R} \sum_{g=1}^{G} x_{i j k r g} \leq \tilde{e}_{k}, k=1 \ldots K, \\
x_{i j k r g} \geq 0, \forall i, j, k, r, g .
\end{array}\right.
$$

5.2 Expected value method for Model 2

Equivalent deterministic model after applying the properties of Expected value method is given in (15).

5.3 Expected value method for Model 3

Model 3's equivalent deterministic form after applying the properties of Expected value method is given in (16). 


$$
\left\{\begin{array}{l}
\operatorname{Max} \tilde{Z}_{1}=\mathrm{E}\left[\sum_{i=1}^{m} \sum_{j=1}^{n} \sum_{k=1}^{K} \sum_{r=1}^{R} \sum_{g=1}^{G}\left[\tilde{S}_{j g}\left(1-\beta_{i j k r g} * d_{i j r}\right)-\tilde{P}_{i g}-\left(\tilde{c}_{i j k r g} * d_{i j r}\right)\right] * x_{i j k r g}\right] \\
\operatorname{Min} \tilde{Z}_{2}=\mathrm{E}\left[\sum_{i=1}^{m} \sum_{j=1}^{n} \sum_{k=1}^{K} \sum_{r=1}^{R} \frac{d_{i j r} * y_{i j k r}}{v_{k}\left(1-\gamma_{i j r}\right)}+\sum_{i=1}^{m} \sum_{j=1}^{n} \sum_{k=1}^{K} \sum_{r=1}^{R} \sum_{g=1}^{G} \tilde{\delta}_{i j k g} * x_{i j k r g}\right] \\
\text { where, } \quad y_{i j k r}\left\{\begin{array}{l}
1, \text { if } \sum_{g=1}^{G} x_{i j k r g}>0, \\
0, \text { if } \sum_{g=1}^{G} x_{i j k r g}=0
\end{array}\right. \\
\text { subject } \quad \text { to, } \\
\mathrm{E}\left[\sum_{j=1}^{n} \sum_{k=1}^{K} \sum_{r=1}^{R} x_{i j k r g}-\tilde{a}_{i g}\right] \leq 0, i=1 \ldots m, g=1 \ldots G, \\
\mathrm{E}\left[\tilde{b}_{j g}-\sum_{i=1}^{m} \sum_{k=1}^{K} \sum_{r=1}^{R}\left(1-\left(\beta_{i j k r g} * d_{i j r}\right)\right) x_{i j k r g}\right] \leq 0, j=1 \ldots n, g=1 \ldots G \\
\mathrm{E}\left[\sum_{i=1}^{m} \sum_{j=1}^{n} \sum_{r=1}^{R} \sum_{g=1}^{G} x_{i j k r g}-\tilde{e}_{k}\right] \leq 0, k=1 \ldots K, \\
x_{i j k r g} \geq 0, \forall i, j, k, r, g .
\end{array}\right.
$$

Definition 9 A feasible solution $X^{*}=\left\{x_{i j k r g}^{*}\right\} \in S$ is an efficient (no dominated) solution for MOMI4DTP with vehicle speed iff there does not exist another $X=\left\{x_{i j k r g}\right\} \in S_{\text {such }}$ that $Z_{k}(X) \leq Z_{k}\left(X^{*}\right), 1 \leq k \leq K$ and $Z_{l}(X) \neq Z_{l}\left(X^{*}\right)$ for some $l, 1 \leq l \leq K$. 


$$
\left\{\begin{array}{l}
\operatorname{Max} Z_{1}=\sum_{i=1}^{m} \sum_{j=1}^{n} \sum_{k=1}^{K} \sum_{r=1}^{R} \sum_{g=1}^{G}\left[E\left[\tilde{S}_{j g}\right]\left(1-\beta_{i j k r g} * d_{i j r}\right)-E\left[\tilde{P}_{i g}\right]-E\left[\left(\tilde{c}_{i j k r g} * d_{i j r}\right)\right]\right] * x_{i j k r g} \\
\operatorname{Min} Z_{2}=\sum_{i=1}^{m} \sum_{j=1}^{n} \sum_{k=1}^{K} \sum_{r=1}^{R} \frac{d_{i j r} * y_{i j k r}}{v_{k}\left(1-\gamma_{i j r}\right)}+\sum_{i=1}^{m} \sum_{j=1}^{n} \sum_{k=1}^{K} \sum_{r=1}^{R} \sum_{g=1}^{G} E\left[\tilde{\delta}_{i j k g}\right] * x_{i j k r g} \\
\text { where, } \quad y_{i j k r}=\left\{\begin{array}{l}
1, i \mathrm{f} \sum_{g=1}^{G} x_{i j k r g}>0, \\
0, i f \sum_{g=1}^{G} x_{i j k r g}=0
\end{array}\right. \\
\text { subject to, } \\
\sum_{j=1}^{n} \sum_{k=1}^{K} \sum_{r=1}^{R} x_{i j k r g}-E\left[\tilde{a}_{i g}\right] \leq 0, i=1 \ldots m, g=1 \ldots G, \\
E\left[\tilde{b}_{j g}\right]-\sum_{i=1}^{m} \sum_{k=1}^{K} \sum_{r=1}^{R}\left(1-\left(\beta_{i j k r g} * d_{i j r}\right)\right) x_{i j k r g} \leq 0, j=1 \ldots n, g=1 \ldots . . G \\
\sum_{i=1}^{m} \sum_{j=1}^{n} \sum_{r=1}^{R} \sum_{g=1}^{G} x_{i j k r g}-E\left[\tilde{e}_{k}\right] \leq 0, k=1 \ldots K, \\
x_{i j k r g} \geq 0, \forall i, j, k, r, g .
\end{array}\right.
$$

Definition 10 A feasible solution $X^{*}=\left\{x_{i j k r g}^{*}\right\} \in S$ is a week efficient solution for multi objective multi item four dimensional transportation problem with vehicle speed iff there does not exist another $X=\left\{x_{i j k r g}\right\} \in S_{\text {such that }} Z_{k}(X) \leq Z_{k}\left(X^{*}\right)$ for $\forall k$. 


$$
\left\{\begin{array}{l}
\operatorname{Max} Z_{1}=\sum_{i=1}^{m} \sum_{j=1}^{n} \sum_{k=1}^{K} \sum_{r=1}^{R} \sum_{g=1}^{G}\left[E\left[\tilde{S}_{j g}\right]\left(1-\beta_{g} * d_{i j r}\right)-E\left[\tilde{P}_{i g}\right]-E\left[\left(\tilde{c}_{i j k r g} * d_{i j r}\right)\right]\right] * x_{i j k r g} \\
\operatorname{Min} Z_{2}=\sum_{i=1}^{m} \sum_{j=1}^{n} \sum_{k=1}^{K} \sum_{r=1}^{R} \frac{d_{i j r} * y_{i j k r}}{v_{k}\left(1-\gamma_{i j r}\right)}+\sum_{i=1}^{m} \sum_{j=1}^{n} \sum_{k=1}^{K} \sum_{r=1}^{R} \sum_{g=1}^{G} E\left[\tilde{\delta}_{i j k g} *^{*} x_{i j k r g}\right. \\
\text { where, } \quad y_{i j k r}=\left\{\begin{array}{l}
1, i \mathrm{f} \sum_{g=1}^{G} x_{i j k r g}>0, \\
0, i f \sum_{g=1}^{G} x_{i j k r g}=0
\end{array}\right. \\
\text { subject } \quad \text { to, } \\
E\left[\tilde{b}_{j g}\right]-\sum_{i=1}^{m} \sum_{k=1}^{K} \sum_{r=1}^{R}\left(1-\left(\beta_{g} * d_{i j r}\right)\right) x_{i j k r g} \leq 0, \forall j, g \\
\sum_{j=1}^{n} \sum_{k=1}^{K} \sum_{r=1}^{R} x_{i j k r g}-E\left[\tilde{a}_{i g}\right] \leq 0, i=1 \ldots m, g=1 \ldots G \\
\sum_{i=1}^{m} \sum_{j=1}^{n} \sum_{r=1}^{R} \sum_{g=1}^{G} x_{i j k r g}-E\left[\tilde{e}_{k}\right] \leq 0, k=1 \ldots K \\
x_{i j k r g} \geq 0, \forall i, j, k, r, g .
\end{array}\right.
$$

$$
\left\{\begin{array}{l}
\operatorname{Max} Z_{1}=\sum_{i=1}^{m} \sum_{j=1}^{n} \sum_{k=1}^{K} \sum_{r=1}^{R} \sum_{g=1}^{G}\left[E\left[\tilde{S}_{j g}\right]-E\left[\tilde{P}_{i g}\right]-E\left[\left(\tilde{c}_{i j k r g} * d_{i j r}\right)\right]\right]^{*} x_{i j k r g} \\
\operatorname{Min} Z_{2}=\sum_{i=1}^{m} \sum_{j=1}^{n} \sum_{k=1}^{K} \sum_{r=1}^{R} \frac{d_{i j r} * y_{i j k r}}{v_{k}\left(1-\gamma_{i j r}\right)}+\sum_{i=1}^{m} \sum_{j=1}^{n} \sum_{k=1}^{K} \sum_{r=1}^{R} \sum_{g=1}^{G} E\left[\tilde{\delta}_{i j k g}\right] * x_{i j k r g} \\
\text { where, } y_{i j k r}=\left\{\begin{array}{l}
1, i \mathrm{f} \sum_{g=1}^{G} x_{i j k r g}>0, \\
0, i f \sum_{g=1}^{G} x_{i j k r g}=0
\end{array}\right.
\end{array}\right.
$$

subject to,

$E\left[\tilde{b}_{j g}\right]-\sum_{i=1}^{m} \sum_{k=1}^{K} \sum_{r=1}^{R} x_{i j k r g} \leq 0 \forall j, g$

$\sum_{j=1}^{n} \sum_{k=1}^{K} \sum_{r=1}^{R} x_{i j k r g}-E\left[\tilde{a}_{i g}\right] \leq 0, i=1 \ldots m, g=1 \ldots G$

$\sum_{i=1}^{m} \sum_{j=1}^{n} \sum_{r=1}^{R} \sum_{g=1}^{G} x_{i j k r g}-E\left[\tilde{e}_{k}\right] \leq 0, k=1 \ldots K$
$x_{i j k r g} \geq 0, \forall i, j, k, r, g$. 


\section{Chance Constrained Method For UMOMI4DTPS}

In this section, we have introduced the equivalent deterministic model for UMOMI4DTPS using chance constrained method.

6.1 Chance constraint method for Model 1

Suppose that $\tilde{S}_{j g}, \tilde{P}_{i g}, \tilde{c}_{i j k r g}, \tilde{\delta}_{i j k g}, \tilde{a}_{i g}, \tilde{b}_{j g}$ and $\tilde{e}_{k}$ are autonomousindeterminate variables with regular indecision distribution $\chi_{j g}, \phi_{i g}, \varphi_{i j k r g}, \theta_{i j k g}, \psi_{i g}, \kappa_{j g}, \lambda_{k} \quad$ respectively. Model 1's equivalent deterministic model using the chance constrained method is given in (17).

6.2 Chance constraint method for Model 2

Equivalent deterministic form of Model 2 using the chance constraint method is given in (18).

6.3 Chance constraint method for Model 3

Model 3's equivalent deterministic form using the chance constraint model is given in (19).

$$
\left\{\begin{array}{l}
\operatorname{Max} Z_{1}^{*}=\sum_{i=1}^{m} \sum_{j=1}^{n} \sum_{k=1}^{K} \sum_{r=1}^{R} \sum_{g=1}^{G}\left[\chi_{j g}^{-1}\left(\alpha_{1}\right)\left(1-\beta_{i j k r g} * d_{i j r}\right)-\phi_{i g}^{-1}\left(\alpha_{2}\right)-\left(\varphi_{i j k r g}^{-1}\left(\alpha_{3}\right) * d_{i j r}\right)\right] x_{i j k r g} \\
\operatorname{Min} Z_{2}^{*}=\sum_{i=1}^{m} \sum_{j=1}^{n} \sum_{k=1}^{K} \sum_{r=1}^{R} \frac{d_{i j r} * y_{i j k r}}{v_{k}\left(1-\gamma_{i j r}\right)}+\sum_{i=1}^{m} \sum_{j=1}^{n} \sum_{k=1}^{K} \sum_{r=1}^{R} \sum_{g=1}^{G} \theta_{i j k g}^{-1}\left(\alpha_{4}\right) * x_{i j k r g} \\
\text { where, } y_{i j k r}=\left\{\begin{array}{l}
1, i \mathrm{f} \sum_{g=1}^{G} x_{i j k r g}>0, \\
0, i \mathrm{f} \sum_{g=1}^{G} x_{i j k r g}=0
\end{array}\right. \\
\begin{array}{l}
\operatorname{subject}^{n} \quad \text { to, } \\
\sum_{j=1}^{n} \sum_{k=1}^{K} \sum_{r=1}^{R} x_{i j k r g} \leq \psi \psi_{i g}^{-1}\left(1-\alpha_{5}\right), i=1 \ldots m, g=1 \ldots G \\
\sum_{i=1}^{m} \sum_{k=1}^{K} \sum_{r=1}^{R}\left(1-\beta_{i j k r g} * d_{i j r}\right) x_{i j k r g} \geq \kappa_{j g}^{-1}\left[\alpha_{6}\right], j=1 \ldots n, g=1 \ldots G \\
\sum_{i=1}^{m} \sum_{j=1}^{n} \sum_{r=1}^{R} \sum_{g=1}^{G} x_{i j k r g} \leq \lambda_{k}^{-1}\left[1-\alpha_{7}\right], k=1 \ldots K \\
x_{i j k r g} \geq 0, \forall i, j, k, r, g .
\end{array}
\end{array}\right.
$$

Where $\alpha_{1}, \alpha_{2}, \alpha_{3}, \alpha_{4}, \alpha_{5}, \alpha_{6}$ and $\alpha_{7}$ are predetermined confidence level and $\alpha_{i} \in(0,1), i=1,2, \ldots 7$. 


$$
\left\{\begin{array}{l}
\operatorname{Max} Z_{1}^{*}=\sum_{i=1}^{m} \sum_{j=1}^{n} \sum_{k=1}^{K} \sum_{r=1}^{R} \sum_{g=1}^{G}\left[\chi_{j g}^{-1}\left(\alpha_{1}\right)\left(1-\beta_{g}^{*} d_{i j r}\right)-\phi_{i g}^{-1}\left(\alpha_{2}\right)-\left(\varphi_{i j k r g}^{-1}\left(\alpha_{3}\right) * d_{i j r}\right)\right] * x_{i j k r g} \\
\operatorname{Min} Z_{2}^{*}=\sum_{i=1}^{m} \sum_{j=1}^{n} \sum_{k=1}^{K} \sum_{r=1}^{R} \frac{d_{i j r} * y_{i j k r}}{v_{k}\left(1-\gamma_{i j r}\right)}+\sum_{i=1}^{m} \sum_{j=1}^{n} \sum_{k=1}^{K} \sum_{r=1}^{R} \sum_{g=1}^{G} \theta_{i j k g}^{-1}\left(\alpha_{4}\right) * x_{i j k r g} \\
\text { where, } \quad y_{i j k r}=\left\{\begin{array}{l}
1, i f \sum_{g=1}^{G} x_{i j k r g}>0, \\
0, i f \sum_{g=1}^{G} x_{i j k r g}=0
\end{array}\right. \\
\text { subject to } \\
\sum_{i=1}^{m} \sum_{k=1}^{K} \sum_{r=1}^{R}\left(1-\left(\beta_{g} * d_{i j r}\right)\right) x_{i j k r g} \geq \kappa_{j g}^{-1}\left[\alpha_{6}\right], j=1 \ldots n, g=1 \ldots G \\
\sum_{j=1}^{n} \sum_{k=1}^{K} \sum_{r=1}^{R} x_{i j k r g} \leq \psi_{i g}^{-1}\left(1-\alpha_{5}\right), i=1 \ldots m, g=1 \ldots G \\
\sum_{i=1}^{m} \sum_{j=1}^{n} \sum_{r=1}^{R} \sum_{g=1}^{G} x_{i j k r g} \leq \lambda_{k}^{-1}\left[1-\alpha_{7}\right], k=1 \ldots K \\
x_{i j k r g} \geq 0, \forall i, j, k, r, g .
\end{array}\right.
$$

Where $\alpha_{1}, \ldots, \alpha_{6}$ and $\alpha_{7}$ are predetermined confidence level and $\alpha_{i} \in(0,1), i=1,2, \ldots 7$.

$$
\begin{aligned}
& \operatorname{Max} Z_{1}^{*}=\sum_{i=1}^{m} \sum_{j=1}^{n} \sum_{k=1}^{K} \sum_{r=1}^{R} \sum_{g=1}^{G}\left[\psi_{j g}^{-1}\left(\alpha_{1}\right)-\phi_{i g}^{-1}\left(\alpha_{2}\right)-\left(\varphi_{i j k r g}^{-1}\left(\alpha_{3}\right) * d_{i j r}\right)\right] * x_{i j k r g} \\
& \operatorname{Min} Z_{2}^{*}=\sum_{i=1}^{m} \sum_{j=1}^{n} \sum_{k=1}^{K} \sum_{r=1}^{R} \frac{d_{i j r} * y_{i j k r}}{v_{k}\left(1-\gamma_{i j r}\right)}+\sum_{i=1}^{m} \sum_{j=1}^{n} \sum_{k=1}^{K} \sum_{r=1}^{R} \sum_{g=1}^{G} \theta_{i j k g}^{-1}\left(\alpha_{4}\right) * x_{i j k r g} \\
& \text { where, } \quad y_{i j k r}=\left\{\begin{array}{l}
1, \text { if } \sum_{g=1}^{G} x_{i j k r g}>0, \\
0, i f \sum_{g=1}^{G} x_{i j k r g}=0
\end{array}\right.
\end{aligned}
$$

subject to,

$$
\begin{aligned}
& \sum_{i=1}^{m} \sum_{k=1}^{K} \sum_{r=1}^{R} x_{i j k r g} \geq \kappa_{j g}^{-1}\left[\alpha_{6}\right], j=1 \ldots n, g=1 \ldots G \\
& \sum_{j=1}^{n} \sum_{k=1}^{K} \sum_{r=1}^{R} x_{i j k r g} \leq \psi_{i g}^{-1}\left(1-\alpha_{5}\right), i=1 \ldots m, g=1 \ldots G \\
& \sum_{i=1}^{m} \sum_{j=1}^{n} \sum_{r=1}^{R} \sum_{g=1}^{G} x_{i j k r g} \leq \lambda_{k}^{-1}\left[1-\alpha_{7}\right], k=1 \ldots K \\
& x_{i j k r g} \geq 0, \forall i, j, k, r, g .
\end{aligned}
$$


Where $\alpha_{1}, \ldots, \alpha_{6}$ and $\alpha_{7}$ are predetermined confidence level and $\alpha_{i} \in(0,1), i=1,2, \ldots 7$.

\section{Goal Programming Approach}

The goal programming is a technique proposed by Charnes clan cooper[36] for obtaining satisfactory solution even in the presence of more than one goals. This field was further developed by many authors like T.Chang[37], Pal[38],etc. Fuzzy goal programming approach was introduced by Mohammed[39] for solving MOTP. The same technique was implemented to solve MOTPwith linear membership function as well as non linear membership functions by M.Zangiabadi[40],[41]. Minimizing the distance between $Z=\left(Z_{1}, \ldots, Z_{w}\right)$ and aspiration (or) target level $\bar{Z}=\left(\bar{Z}_{1}, \ldots, \bar{Z}_{w}\right)$,which are set by the decision maker, is the purpose of goal programming. For this purpose, the negative and positive deviational variables have been introduced below.

$$
\begin{aligned}
& D_{w}^{+}=\max \left(0, Z_{w}-\bar{Z}_{w}\right) \\
& D_{w}^{-}=\max \left(0, \bar{Z}_{r}-Z_{w}\right)
\end{aligned}
$$

To minimize the distance between $Z_{w}$ and $\bar{Z}_{w}$, we have to minimize either $D_{w}^{+}, D_{w}^{-}$or $D_{w}^{+}+D_{w}^{-}$. When we have to maximize $Z_{w}, g_{w}\left(D_{w}^{+}, D_{w}^{-}\right)=D_{w}^{-}$. While, when we have to $\operatorname{minimize} Z_{w}, g_{w}\left(D_{w}^{+}, D_{w}^{-}\right)=D_{w}^{+}$. When we desire $Z_{w}=\bar{Z}_{w}, g_{w}\left(D_{w}^{+}, D_{w}^{-}\right)=D_{w}^{-}+D_{w}^{-}$.

Membership functions are defined in order to express the satisfaction of decision maker along with the solution. Given below are the definitions of membership functions.

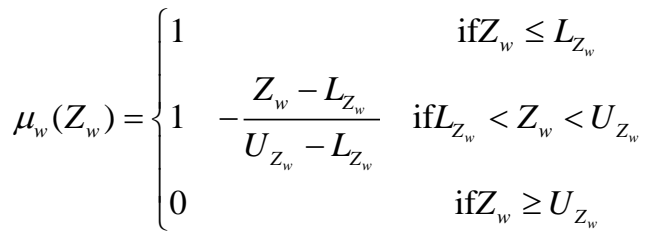

$\mu_{w}\left(Z_{w}\right)$ represents the decision maker's satisfaction. Therefore, it must be maximized i.e $\max \left(\mu_{1}\left(Z_{1}(x)\right), \ldots, \mu_{w}\left(Z_{w}(x)\right)\right)$

Here $U_{Z_{w}}$ and $L_{Z_{w}}$ are the highest acceptable and aspired level of achievement for $Z_{w}$, $(\mathrm{w}=1 ; 2)$ objective function.

We know that the maximum value of any membership function is one. Therefore, to maximize any of the membership functions, we minimize its negative deviation from 1 to make them as close as possible to 1 . The LPP can be formulated as below.

$$
\begin{aligned}
& \min \left(\max \left(g_{w}\left(D_{w}^{+}, D_{W}^{-}\right)\right)\right) \\
& \text {i.e min Q } \\
& \text { subject to } \\
& \frac{U_{Z_{w}}-Z_{w}}{U_{Z_{w}}-L_{Z_{w}}}+D_{w}^{-}-D_{w}^{+}=1,
\end{aligned}
$$


$Q \geq D_{w}^{-}$, where $\mathrm{w}=1 ; 2$.

$D_{w}^{-} \cdot D_{w}^{+}=0$,

$D_{w}^{-}, D_{w}^{+} \geq 0,0 \leq Q \leq 1$, and with given constraints. Here, we have considered UMOMI4DTPS type of problem; goal programming technique will be the most appropriate procedure for getting the most suitable compromise solution.

\section{Algorithm For Solving UMOMI4DTPS}

The following proposed algorithm is used to solve three different models of UMOMI4DTPS.

Step 1: Convert the uncertain MOMI4DTPS into deterministic model by applying the expected value and chance constraint models.

Step 2: Solve the profit and time objective function $Z_{w},(w=1 ; 2)$ individually subject to the supply, demand constraints including breakability and conveyance constraints.

Step 3: Compute the values of every of the objectives function $Z_{w},(w=1 ; 2)$ at each solution obtain in step 2 .

Step 4: From the set of solutions calculated from the step 2, obtained the upper $U_{Z_{w}}$ and lower $L_{Z_{w}}$ bound for each objective function. Here $U_{Z_{w}}$ and $L_{Z_{w}}$ are the highest satisfactory and aspired level of accomplishment for $Z_{w}^{\text {th }},(\mathrm{w}=1 ; 2)$ objective function.

Step 5: Use goal programming technique to formulate the following LPP model for the given UMOMI4DTPS.

Min Q

subject to

$$
\frac{U_{z_{w}}-Z_{w}}{U_{Z_{w}}-L_{Z_{w}}}+D_{w}^{-}-D_{w}^{+}=1
$$

$Q \geq D_{w}^{-}$, where $\mathrm{w}=1,2$.

$D_{w}^{-} \cdot D_{w}^{+}=0$

$D_{w}^{+} . D_{w}^{-} \geq_{0}, 0 \leq Q \leq 1$, and with constraints given in the respective model.

Step 6: Solve the above model obtained in step 4 by using generalized abridged gradient method (LINGO-18.0 Suite Solver) to get the compromise solution.

\section{Numerical Example}

Numerical example of UMOMI4DTPS is presented in this section, whose parameters are linear uncertain variables, to showcase the effectiveness and efficiency of the presented methodology. Here, variables like availabilities, demands, purchase price, selling price, unloading and loading time and transportation cost are considered as indeterminate variables. 
We consider two different sources (origins), customers (destinations) conveyances, roads and different goods each.

i.e $\mathrm{m}=\mathrm{n}=\mathrm{K}=\mathrm{Q}=\mathrm{G}=2$.

Table 1 contains the data for availabilities of goods in the sources.

Table 1: Available stock in each origin

\begin{tabular}{|l|l|l|}
\hline$i$ & $\boldsymbol{g}$ & $\tilde{\boldsymbol{a}}_{i g}$ \\
\hline \multirow{2}{*}{1} & 1 & $\mathrm{~L}(380,400)$ \\
\cline { 2 - 3 } & 2 & $\mathrm{~L}(260,360)$ \\
\hline \multirow{2}{*}{2} & 1 & $\mathrm{~L}(180,380)$ \\
\cline { 2 - 3 } & 2 & $\mathrm{~L}(217,377)$ \\
\hline
\end{tabular}

The following table contains the data for demands of goods in the destination.

Table 2: Demands in the destinations

\begin{tabular}{|l|l|l|}
\hline \multirow{2}{*}{$1.1 j$} & $1.2{ }^{g}$ & $1.3 \tilde{b}_{j g}$ \\
\hline 1.41 & 1.51 & $1.6 \mathrm{~L}(100,164.084)$ \\
\cline { 2 - 3 } & 1.72 & $1.8 \mathrm{~L}(50,150)$ \\
\hline \multirow{2}{*}{1.92} & 1.101 & $1.11 \mathrm{~L}(\mathbf{7 0 , 1 0 0 )}$ \\
\cline { 2 - 3 } & 1.122 & $1.13 \mathrm{~L}(110,130)$ \\
\hline
\end{tabular}

The purchasing prices of the goods are given in table 3 .

Table 3: Purchasing price for the goods

\begin{tabular}{|l|l|l|}
\hline $1.14 i$ & $1.15{ }^{g}$ & $\tilde{P}_{i g}$ \\
\hline \multirow{2}{*}{1.171} & 1.181 & $1.19 \mathrm{~L}(8.250,20)$ \\
\cline { 2 - 3 } & 1.202 & $1.21 \mathrm{~L}(12,14)$ \\
\hline \multirow{2}{*}{1.222} & 1.231 & $1.24 \mathrm{~L}(7,15)$ \\
\cline { 2 - 3 } & 1.252 & $1.26 \mathrm{~L}(11,13)$ \\
\hline
\end{tabular}

The selling prices of ${ }^{g^{\text {th }}}$ goods in the $j^{\text {th }}$ destinations are in the following table.

Table 4: Selling price for the goods

\begin{tabular}{|l|l|l|}
\hline $1.27 j$ & 1.28 & $\tilde{S}_{i g}$ \\
\hline 1.301 & 1.311 & $1.32 \mathrm{~L}(\mathbf{7 0 , 1 0 4 )}$ \\
\cline { 2 - 3 } & 1.332 & $1.34 \mathrm{~L}(110,120)$ \\
\hline 1.352 & 1.361 & $1.37 \mathrm{~L}(100,124)$ \\
\cline { 2 - 3 } & 1.382 & $1.39 \mathrm{~L}(100,104)$ \\
\hline
\end{tabular}

Table 5 contains the data for capacity of the transports.

\begin{tabular}{|c|c|}
\hline $1.40 \widetilde{\boldsymbol{e}}_{1}$ & $1.41 \widetilde{\boldsymbol{e}}_{2}$ \\
\hline $1.42 \mathrm{~L}(300,496)$ & $1.43 \mathrm{~L}(330,500)$ \\
\hline
\end{tabular}

The loading and unloading time of different goods are presented in table 6 .

Table 6: loading and unloading timing

\begin{tabular}{|l|l|l|l|l|}
\hline $\boldsymbol{i}$ & $j$ & $\boldsymbol{k}$ & $\tilde{\delta}_{i j k 1}$ & $\tilde{\delta}_{i j k 2}$ \\
\hline 1 & 1 & 1 & $\mathrm{~L}(.3, .5)$ & $\mathrm{L}(.3, .5)$ \\
\hline
\end{tabular}




\begin{tabular}{|l|l|l|l|l|} 
& & 2 & $\mathrm{~L}(.3, .8)$ & $\mathrm{L}(.2, .5)$ \\
\cline { 2 - 5 } & 2 & 1 & $\mathrm{~L}(.5, .7)$ & $\mathrm{L}(.34, .5)$ \\
\cline { 3 - 5 } & & 2 & $\mathrm{~L}(.22, .60)$ & $\mathrm{L}(.22, .60)$ \\
\hline \multirow{3}{*}{2} & \multirow{2}{*}{1} & 1 & $\mathrm{~L}(.56, .7)$ & $\mathrm{L}(.6, .8)$ \\
\cline { 3 - 5 } & & 2 & $\mathrm{~L}(.3, .7)$ & $\mathrm{L}(.8,1)$ \\
\cline { 2 - 5 } & \multirow{2}{*}{2} & 1 & $\mathrm{~L}(.8,1)$ & $\mathrm{L}(.1, .5)$ \\
\cline { 3 - 5 } & & 2 & $\mathrm{~L}(.32, .5)$ & $\mathrm{L}(.34, .5)$ \\
\hline
\end{tabular}

The unit transportation cost of various goods per units distance are in table 7 . The breakability rates of goods per unit distance are given un table 8.

Table 7: Unit transportation cost of different goods per unit distance

\begin{tabular}{|c|c|c|c|c|c|}
\hline$i$ & $j$ & $k$ & $r^{\prime}$ & $\tilde{\boldsymbol{c}}_{i j k r 1}$ & $\tilde{\boldsymbol{c}}_{i j k r 2}$ \\
\hline \multirow[t]{8}{*}{1} & \multirow[t]{4}{*}{1} & \multirow[t]{2}{*}{1} & 1 & $\mathrm{~L}(.20, .48)$ & $\mathrm{L}(.20, .48)$ \\
\hline & & & 2 & $\mathrm{~L}(.32, .40)$ & $\mathrm{L}(.20, .40)$ \\
\hline & & \multirow[t]{2}{*}{2} & 1 & $\mathrm{~L}(.26,40)$ & $\mathrm{L}(.16,30)$ \\
\hline & & & 2 & $\mathrm{~L}(.40, .57)$ & $\mathrm{L}(.1, .3)$ \\
\hline & \multirow[t]{4}{*}{2} & \multirow[t]{2}{*}{1} & 1 & $\mathrm{~L}(.30, .56)$ & $\mathrm{L}(.12, .30)$ \\
\hline & & & 2 & $\mathrm{~L}(.34, .50)$ & $\mathrm{L}(.22, .30)$ \\
\hline & & \multirow[t]{2}{*}{2} & 1 & $\mathrm{~L}(.2, .6)$ & $\mathrm{L}(.28, .40)$ \\
\hline & & & 2 & $\mathrm{~L}(.3, .7)$ & $\mathrm{L}(.34, .60)$ \\
\hline \multirow[t]{8}{*}{2} & \multirow[t]{4}{*}{1} & \multirow[t]{2}{*}{1} & 1 & $\mathrm{~L}(.38, .50)$ & $\mathrm{L}(.200, .270)$ \\
\hline & & & 2 & $\mathrm{~L}(.40, .52)$ & $\mathrm{L}(.12, .40)$ \\
\hline & & \multirow[t]{2}{*}{2} & 1 & $\mathrm{~L}(.2,6)$ & $\mathrm{L}(.2,4)$ \\
\hline & & & 2 & $\mathrm{~L}(.40, .56)$ & $\mathrm{L}(.30, .66)$ \\
\hline & \multirow[t]{4}{*}{2} & \multirow[t]{2}{*}{1} & 1 & $\mathrm{~L}(.340, .500)$ & $\mathrm{L}(.200, .012)$ \\
\hline & & & 2 & $\mathrm{~L}(.260, .700)$ & $\mathrm{L}(.1, .34)$ \\
\hline & & \multirow[t]{2}{*}{2} & 1 & $\mathrm{~L}(.400, .410)$ & $\mathrm{L}(.2,4)$ \\
\hline & & & 2 & $\mathrm{~L}(.3,7)$ & $\mathrm{L}(.1,3)$ \\
\hline
\end{tabular}

Table 8: Rate of breakability per unit distance

\begin{tabular}{|c|c|c|c|c|c|}
\hline$i$ & $j$ & $k$ & $r$ & $\beta_{i j k r 1}$ & $\beta_{i j k r 2}$ \\
\hline \multirow[t]{8}{*}{1} & \multirow[t]{4}{*}{1} & \multirow[t]{2}{*}{1} & 1 & 0.014 & 0.024 \\
\hline & & & 2 & 0.014 & 0.015 \\
\hline & & \multirow[t]{2}{*}{2} & 1 & 0.009 & 0.01 \\
\hline & & & 2 & 0.024 & 0.014 \\
\hline & \multirow[t]{4}{*}{2} & \multirow[t]{2}{*}{1} & 1 & 0.015 & 0.025 \\
\hline & & & 2 & 0.024 & 0.015 \\
\hline & & \multirow[t]{2}{*}{2} & 1 & 0.014 & 0.015 \\
\hline & & & 2 & 0.011 & 0.012 \\
\hline \multirow[t]{4}{*}{2} & \multirow[t]{4}{*}{1} & \multirow[t]{2}{*}{1} & 1 & 0.014 & 0.024 \\
\hline & & & 2 & 0.024 & 0.024 \\
\hline & & \multirow[t]{2}{*}{2} & 1 & 0.019 & 0.019 \\
\hline & & & 2 & 0.016 & 0.009 \\
\hline
\end{tabular}




\begin{tabular}{|l|l|l|l|l|}
2 & 1 & 1 & 0.012 & 0.016 \\
\cline { 3 - 5 } & & 2 & 0.014 & 0.01 \\
\cline { 2 - 5 } & 2 & 1 & 0.012 & 0.016 \\
\cline { 3 - 5 } & & 2 & 0.012 & 0.011 \\
\hline
\end{tabular}

The speed of different transports are in the following table 9.

Table 9: Speed of different transport

\begin{tabular}{|l|l|}
\hline $1.44 \mathrm{~V}_{1}$ & $1.45 \mathrm{~V}_{2}$ \\
\hline 1.4635 & 1.4725 \\
\hline
\end{tabular}

Distance amongdissimilar sources and destination via dissimilar route are assumed by table 10 .

Table 10: Distance from source to destination via different routes

\begin{tabular}{|l|l|l|l|}
\hline \multirow{2}{*}{$\boldsymbol{i}$} & $j$ & $\boldsymbol{d}_{\boldsymbol{i} 1}$ & $\boldsymbol{d}_{\boldsymbol{i} j 2}$ \\
\hline \multirow{2}{*}{1} & 1 & 33 & 45 \\
\cline { 2 - 4 } & 2 & 45 & 35 \\
\hline \multirow{2}{*}{2} & 1 & 43 & 40 \\
\cline { 2 - 4 } & 2 & 56 & 45 \\
\hline
\end{tabular}

Table 11 contains the data of rate of disturbance of speeds.

Table 11: Rate of disturbance

\begin{tabular}{|l|l|l|l|}
\hline \multirow{i}{*}{$\boldsymbol{i}$} & $j$ & $\gamma_{i j 1}$ & $\gamma_{i j 2}$ \\
\hline \multirow{3}{*}{1} & 1 & 0.0122 & 0.03 \\
\cline { 2 - 4 } & 2 & 0.013 & 0.022 \\
\hline \multirow{2}{*}{2} & 1 & 0.03 & 0.025 \\
\cline { 2 - 4 } & 2 & 0.011 & 0.04 \\
\hline
\end{tabular}

Applying the abovedevelopedalgorithm for the problem taken, the steps are:

Here we have explained the procedure for expected value model 1 alone.

Step 1: For the above data, deterministic optimization problems of the given model is obtained using expected value method(14) and solved.

Step 2: Solving the above objectives separately, we have $Z_{1}=-31077.4$ and $Z_{2}=278.967$ follow:

By using the calculated solutions, the value of each objectivespurpose is obtained as

$Z_{1}\left(x_{1}\right)=-31077.4, Z_{1}\left(x_{2}\right)=-26711.88$

$Z_{2}\left(x_{1}\right)=306.026, Z_{2}\left(x_{2}\right)=278.967$

The upper and lower bounds of each objective functions are as follows:

$U_{z_{1}}=-26711.88, L_{z_{1}}=-31077.4$

$U_{z_{2}}=306.027, L_{z_{2}}=278.967$

Step 3: Using goal programming technique, the goal programming expected value method for Model 1 is expressed as follow. 


$$
\left\{\begin{array}{l}
\text { Min } Q \\
\text { subject to } \\
-26711.88+\sum_{i=1}^{m} \sum_{j=1}^{n} \sum_{k=1}^{K} \sum_{r=1}^{R} \sum_{g=1}^{G}\left[E\left[\tilde{S}_{j g}\right]\left(1-\beta_{i j k r g} * d_{i j r}\right)-E\left[\tilde{P}_{i g}\right]-E\left[\left(\tilde{c}_{i j k r g} * d_{i j r}\right)\right]\right]^{*} x_{i j k r g} \\
+4365.520\left(D_{1}^{-}-D_{1}^{+}\right)=4365.520 \\
306.027-\sum_{i=1}^{m} \sum_{j=1}^{n} \sum_{k=1}^{K} \sum_{r=1}^{R} \frac{d_{i j r} * y_{i j k r}}{v_{k}\left(1-\gamma_{i j r}\right)}+\sum_{i=1}^{m} \sum_{j=1}^{n} \sum_{k=1}^{K} \sum_{r=1}^{R} \sum_{g=1}^{G} E\left[\tilde{\delta}_{i j k g}\right]^{*} x_{i j k r g} \\
+27.060\left(D_{2}^{-}-D_{2}^{+}\right)=27.060 \\
Q \geq D_{w}^{-} \text {where w }=1,2 \\
\mathrm{D}_{w}^{+} \cdot D_{w}^{-} \geq 0,0 \leq Q \leq 1 \\
\text { where, } y_{i j k r}=\left\{\begin{array}{l}
1, i f \sum_{g=1}^{G} x_{i j k r g}>0, \\
0, i f \sum_{g=1}^{G} x_{i j k r g}=0
\end{array}\right. \\
\text { subject to, } \\
\sum_{j=1}^{n} \sum_{k=1}^{K} \sum_{r=1}^{R} x_{i j k r g}-E\left[\tilde{a}_{i g}\right] \leq 0, i=1 \ldots m, g=1 \ldots G \\
E\left[\tilde{b}_{j g}\right]-\sum_{i=1}^{m} \sum_{k=1}^{K} \sum_{r=1}^{R}\left(1-\left(\beta_{i j k r g} * d_{i j r}\right)\right) x_{i j k r g} \leq 0, j=1 \ldots n, g=1 \ldots . . G \\
\sum_{i=1}^{m} \sum_{j=1}^{n} \sum_{r=1}^{R} \sum_{g=1}^{G} x_{i j k r g}-E\left[\tilde{e}_{k}\right] \leq 0, k=1 \ldots K \\
x_{i j k r g} \geq 0, \forall i, j, k, r, g .
\end{array}\right.
$$

Step 4: By using the generalizedabridged gradient method LINGO-18.0 Suite Solver, we obtain the efficient value of $\mathrm{Q}=0: 482$ and the corresponding transportation plan is $D_{1}^{-}=.482, D_{1}^{+}=0, D_{2}^{-}=.482, D_{2}^{+}=0, \operatorname{Max}$ Min $Z_{2}=291.997, x_{11111}=123.008, x_{11211}=93.691$,

$x_{11212}=183.098, x_{12221}=138.211, x_{22122}=218.182, y_{1111}=1, y_{1121}=1, y_{1222}=1, y_{2212}=1 . \quad$ The remaining decision variables values are 0 . We can conclude that both the objectives are achieved with decision maker's satisfaction. Repeating the above steps from 1 to 4 for the remaining two expected value models and chance constraint models, we have the following results.

Table 12 contains the calculated objective values of each objective function (14),(15) and (16) in each model. The efficient solutions of each goal programming expected value models are given in detail in table 13.

Table 12: Objective values of expected value models

\begin{tabular}{|l|l|l|l|l|l|l|}
\hline $\begin{array}{l}\text { Model } \\
\text { no. }\end{array}$ & $\operatorname{Max} Z_{1}$ & $x_{i j k r g}$ & $y_{i j k r}$ & $\operatorname{Min} Z_{2}$ & $x_{i j k r}$ & $y_{i j k r}$ \\
\cline { 1 - 5 } Model 1 & 31077.4 & $x_{11111}=179.82$ & $y_{1111}=1$ & 278.967 & $x_{11111}=179.82$ & $y_{1111}=1$ \\
\cline { 1 - 1 }
\end{tabular}




\begin{tabular}{|c|c|c|c|c|c|c|}
\hline & & $\begin{array}{l}x_{11211}=50.22 \\
x_{11212}=226.57 \\
x_{12221}=138.21 \\
x_{22122}=218.18\end{array}$ & $\begin{array}{l}y_{1121}=1 \\
y_{1222}=1 \\
y_{2212}=1\end{array}$ & & $\begin{array}{l}x_{11211}=50.22 \\
x_{11212}=149.25 \\
x_{12221}=138.21 \\
x_{22122}=218.18\end{array}$ & $\begin{array}{l}y_{1121}=1 \\
y_{1222}=1 \\
y_{2212}=1\end{array}$ \\
\hline 'Model 2 & 43324.36 & $\begin{array}{l}x_{11211}=152.75 \\
x_{11212}=149.26 \\
x_{12121}=237.25 \\
x_{12122}=160.75 \\
x_{21211}=5.73 \\
x_{22221}=79.06 \\
x_{22222}=28.21\end{array}$ & $\begin{array}{l}y_{1121}=1 \\
y_{1212}=1 \\
y_{2121}=1 \\
y_{2222}=1\end{array}$ & 228.25 & $\begin{array}{l}x_{11111}=158.14 \\
x_{11212}=149.25 \\
x_{12221}=103.03 \\
x_{22122}=218.18\end{array}$ & $\begin{array}{l}y_{1111}=1 \\
y_{1121}=1 \\
y_{1222}=1 \\
y_{2212}=1\end{array}$ \\
\hline Model 3 & 70016.99 & $\begin{array}{l}x_{11111}=27.04 \\
x_{11211}=105.00 \\
x_{11212}=310.00 \\
x_{12121}=85.00 \\
x_{21112}=165.96 \\
x_{22112}=120.00\end{array}$ & $\begin{array}{l}y_{1111}=1 \\
y_{1121}=1 \\
y_{1212}=1 \\
y_{2111}=1 \\
y_{2211}=1\end{array}$ & 174.49 & $\begin{array}{l}x_{11111}=115.43 \\
x_{11121}=16.62 \\
x_{11212}=50.00 \\
x_{11222}=50.00 \\
x_{12211}=15.62 \\
x_{12221}=15.62 \\
x_{22112}=77.73 \\
x_{22122}=42.27 \\
x_{22211}=15.62 \\
x_{22221}=38.16\end{array}$ & $\begin{array}{l}y_{1111}=1 \\
y_{1112}=1 \\
y_{1121}=1 \\
y_{1122}=1 \\
y_{1221}=1 \\
y_{1222}=1 \\
y_{2211}=1 \\
y_{2212}=1 \\
y_{2221}=1 \\
y_{2222}=1\end{array}$ \\
\hline
\end{tabular}

Table 13: The efficient solution of Expected value MOMI4DTPS

\begin{tabular}{|c|c|c|c|c|c|c|c|c|}
\hline \multirow{2}{*}{$\begin{array}{l}\text { Model } \\
\text { no. }\end{array}$} & \multirow{2}{*}{$\begin{array}{l}\text { Objective } \\
\text { value }\end{array}$} & \multicolumn{2}{|c|}{$\operatorname{Max} Z_{1}$} & \multicolumn{2}{|c|}{$\operatorname{Min} Z_{1}$} & \multirow{2}{*}{$\operatorname{Max} Z_{1}$} & \multirow{2}{*}{$\begin{array}{c}\operatorname{Min} \\
Z_{1}\end{array}$} & \multirow{2}{*}{ Objective value } \\
\hline & & $D_{w}^{+}$ & $D_{w}^{-}$ & $D_{w}^{+}$ & $D_{w}^{-}$ & & & \\
\hline Model 1 & 0.48 & 0 & 0.48 & 0 & 0.48 & 28975 & 291.99 & $\begin{array}{l}x_{11111}=123.01, x_{11211}=93.69 \\
x_{12221}=138.21, x_{11212}=183.1 \\
x_{22122}=218.18, y_{1111}=1 \\
y_{1121}=y_{1222}=y_{2212}=1\end{array}$ \\
\hline Model 2 & 0.33 & 0 & 0.33 & 0 & 0.33 & 38784.04 & 285.04 & $\begin{array}{l}x_{11111}=158.14, x_{11212}=310 \\
x_{12221}=104.32, x_{22122}=218.18 \\
y_{1111}=y_{1121}=y_{1222}=y_{2212}=1\end{array}$ \\
\hline Model 3 & 0.35 & 0 & 0.35 & 0 & 0.35 & 57575.34 & 247.85 & $\begin{array}{l}x_{11111}=132.04, x_{11212}=214.44 \\
x_{12221}=85, x_{22112}=265.96 \\
y_{1111}=y_{1121}=y_{1222}=y_{2211}=1\end{array}$ \\
\hline
\end{tabular}

By using goal programming technique, the efficient solution for UMOMI4DTPS are obtained as of table 13 . 
Table 14 contains the calculated objective values of each of the chance constraint model objectives(17), (18) and (19), where $\alpha_{1}=0.6, \alpha_{2}=0.9, \alpha_{3}=0.9 . \alpha_{4}=0.9, \alpha_{5}=0.3, \alpha_{6}=0.9, \alpha_{7}=0.3$.

Table 14: Objective values of chance constraint model

\begin{tabular}{|c|c|c|c|c|}
\hline $\begin{array}{l}\text { Model } \\
\text { no. }\end{array}$ & $\operatorname{Max} Z_{1}$ & $\boldsymbol{x}_{i j k r g}$ and $\boldsymbol{y}_{i j k r}$ & $\operatorname{Min} Z_{2}$ & $\boldsymbol{x}_{i j k r g}$ and $\boldsymbol{y}_{i j k r}$ \\
\hline Model 1 & 30706.69 & $\begin{array}{l}x_{11111}=147.96, x_{11211}=111.06 \\
x_{11212}=208.8, x_{12221}=128.98 \\
x_{22121}=47.77, x_{22122}=241.47 \\
y_{1111}=y_{1121}=y_{1222}=y_{2212}=1\end{array}$ & 281.8 & $\begin{array}{l}x_{11111}=203.95, x_{11211}=668.21 \\
x_{11212}=208.95, x_{12221}=115.84 \\
x_{22122}=232.73, x_{22221}=55.99 \\
y_{1111}=y_{1121}=y_{1222}=y_{2222}=1 \\
y_{2212}=1\end{array}$ \\
\hline Model2 & 46675.4 & $\begin{array}{l}x_{11211}=118.72, x_{11212}=207.21 \\
x_{12121}=269.28, x_{12122}=92.79 \\
x_{22121}=73.19, x_{21122}=1.95 \\
x_{22222}=123.07, y_{1121}=y_{1212}=1 \\
y_{2112}=1\end{array}$ & 223.63 & $\begin{array}{l}x_{11111}=188.83, x_{11212}=208.96 \\
x_{12221}=117.58, x_{22122}=232.73 \\
y_{1111}=y_{1121}=1 \\
y_{1222}=y_{2212}=1 \\
y_{2222}=1\end{array}$ \\
\hline Model 3 & 78182.42 & $\begin{array}{l}x_{11111}=8.68, x_{11211}=149 \\
x_{11212}=300, x_{12121}=147.52 \\
x_{21112}=153, x_{22112}=128 \\
y_{1111}=y_{1121}=y_{1212}=y_{2111}=1 \\
y_{2211}=1\end{array}$ & 169.22 & $\begin{array}{l}x_{11111}=135.69, x_{11121}=21.99 \\
x_{11222}=70, x_{11212}=70 \\
x_{12211}=71.79, x_{12221}=25.29 \\
x_{22112}=94.51, x_{22122}=33.50 \\
y_{1111}=y_{1112}=y_{1121}=y_{1122}=1 \\
y_{1221}=y_{1222}=y_{2211}=y_{2212}=1\end{array}$ \\
\hline
\end{tabular}

By using Goal Programming technique, the efficient solution for UMOMI4DTPS are obtained as follows

Table 15: Efficient solution of chance constraint UMOMI4DTPS

\begin{tabular}{|c|c|c|c|c|c|c|c|c|}
\hline \multirow{2}{*}{ Model no. } & \multirow{2}{*}{$\begin{array}{l}\text { Objective } \\
\text { value }\end{array}$} & \multicolumn{2}{|c|}{$\operatorname{Max} Z_{1}$} & \multicolumn{2}{|c|}{$\operatorname{Min} Z_{2}$} & \multirow{2}{*}{$\operatorname{Max} Z_{1}$} & \multirow{2}{*}{$\begin{array}{c}\mathrm{Min} \\
\boldsymbol{Z}_{2}\end{array}$} & \multirow{2}{*}{ Objective value } \\
\hline & & $D_{w}^{+}$ & $D_{w}^{-}$ & $D_{w}^{+}$ & $D_{w}^{-}$ & & & \\
\hline Model 1 & 0.38 & 0 & 0.38 & 0 & 0.38 & 30471.09 & 290.91 & $\begin{array}{l}x_{11111}=164.26, x_{11211}=78.21 \\
x_{12221}=145.53, x_{11212}=208.96 \\
x_{21111}=35.98, x_{22122}=236.96 \\
x_{22222}=16.31, y_{1111}=1 \\
y_{1121}=y_{1222}=y_{2111}=1 \\
y_{2212}=y_{2222}=1\end{array}$ \\
\hline Model 2 & 0.28 & 0 & 0.28 & 0 & 0.28 & 43410.40 & 273.33 & $\begin{array}{l}x_{11111}=209.31, x_{11212}=273.40 \\
x_{12122}=26.60, x_{12221}=175.60 \\
x_{22122}=201.29, y_{1111}=y_{1121}=1 \\
y_{1212}=y_{1222}=y_{2212}=1\end{array}$ \\
\hline
\end{tabular}




\begin{tabular}{|l|l|l|l|l|l|l|l|l|} 
Model 3 & 0.29 & 0 & 0.29 & 0 & 0.29 & 67555.65 & 231.09 & $\begin{array}{l}x_{1111}=157.68, x_{11212}=254.64 \\
x_{12221}=97, x_{22112}=279.52 \\
y_{1111}=y_{1121}=y_{1222}=y_{2211}=1\end{array}$ \\
\hline
\end{tabular}

\section{Discussion}

In our investigation, we have obtained the satisfactory solution of UMOMI4DTPS by GP predictable value and GP coincidental constrained method. The efficient solutions of expected value UMOMI4DTPS and Chance constraint UMOMI4DTPS are obtained using goal programming technique and are given in tables 13 and 15 respectively. Thus the goal programming technique is a fitting technique for dealing multi objectives transportations problems. In Model 1, the breakability of items depend upon different conveyances, items and routes. Where as in Model 2 damage of items which is considered only because of its nature. In Model 3, the damage of items is not considered. We get maximum profit in model 3 as breakability hasn't been considered. In Model 2, there is considerable profit, while in Model1 we get minimal profit as we infer from the table 13. Unlike expected value model where the solution is fixed, in chance constrained model we get optimistic - pessimistic solutions as we can obtain different satisfactory solutions according to condition which is given higher weightage as preferred by the decision maker as inferred from the table 15 .

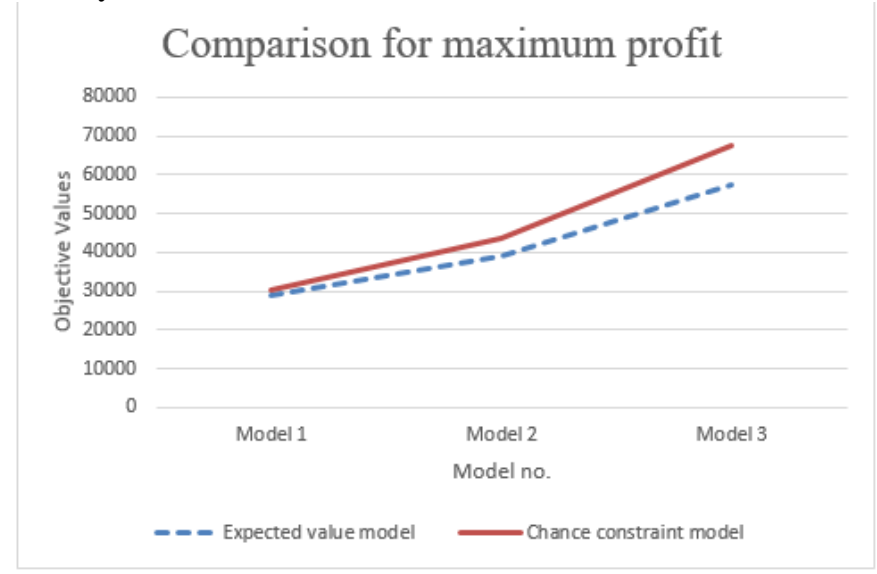

(a) Profit objective function comparison 


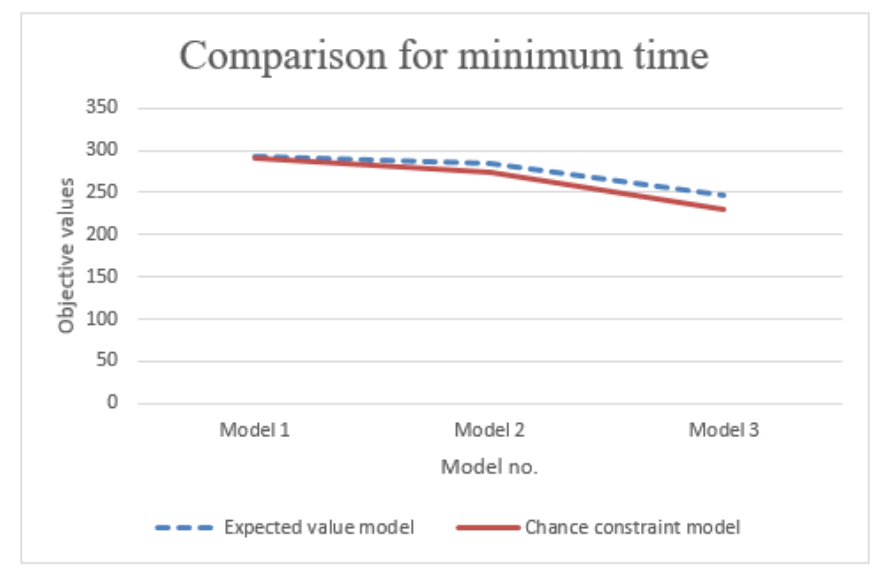

(b) Time objective function comparison

Fig. 1: Graphical analysis

The foremost impartial of this examination is to exploit the turnover and minimalize the transportations period. As we can clearly infer from the graphs (figure 1), the profit obtained in the chance constrained model is higher than of the expected value models. Similarly, the time taken for transportation has also been minimized. Also, the decision maker can obtain solutions according to his preferences which is an added advantage of the proposed method.

\section{Practical Implications}

The presented model can be very much beneficial in real world business applications. Mr.Akash is a television retailer. He deals different types of televisions and has three outlets in Chennai, Puducherry and Madurai. All these products are bought by him from manufacturer located in Tuticorin, Tiruchirappalli and Salem. He uses conveyances (transports) like Lorries, tempos and trucks. There are different paths like NH-7, NH-45, NH-46, NH-68 and other village paths. The distances differ because of the presence of different manufacturer and different outlets. Also, the variety of conveyances may affect the transportation speed. The speed can vary due to the road conditions too. Moreover, televisions are breakable items and the rate of breakability will also vary depending upon the roads. Due to the unpredictable nature of the consumer, the availabilities, prices at different manufacturing companies vary; these parameters are imprecise quantities and are represented through linear uncertain variables. Therefore the present investigation will definitely prove to be useful in real life cases.

\section{Comparision Between Proposed Method And Existing Methods}

The method presented is a type of UMOMI4DTPS with different parameters such as selling price, cost price, availabilities, demands, loading and unloading time, unit transportation cost and the vehicle speed. Till date no method accounted vehicle speed. The proposed method is the first ever model considering vehicle speed .Methodologies and environments to solve MOMISTP have been investigated by many researchers. In 2018, the 
Bera et al[42] has provided a method for MOMI4DTP considering only budget constraints using rough and fuzzy interval environments. However the proposed model is the only model considering all parameters and aspects for the first time. As the proposed model considers diverse parameters, it is beneficial one for the managerial decision making under uncertain environment.

\section{Conclusion}

we have presented a multi objective multi item four dimensional transportation problem vehicle speed under uncertain environment. Unlike other transportation models, here we have considered the variance in vehicle speed because of the road condition. The equivalent deterministic models for UMOMI4DTPS have been obtained by using both expected value and chance constrained methods followed by compromise solution which is obtained by using goal programming technique. The suggested model is identicalinformal to apply, comprehend and is economically more beneficial for a firm as the profit is increased and the transportation time is efficiently minimized too. Hence, the decision maker can make better managerial decisions. The numerical example provided has keenly showed the ease of application of this method as well as provides an efficient solution for UMOMI4DTPS.

\section{References}

[1] F.L.Hitchcock, The distribution of a product from several sources to numerous localities, Journal of Mathematical. Physics, 20 (1941):224-230.

[2] T.C.Koopmans, Optimum utilization of the transportation System, Econometrica, 17 (1949):136146.

[3] Lee, S.M and Moore.L.J, Optimizing transportation problems with multiple objectives. AIEE Transactions, 5(1973): 333- 338.

[4] Pandian.P, Anuradha.D, A new method for solving bi-objective transportation problem. Aust.J. Basic \& Appl. Sci., 10(2011):67-74.

[5] Quddoos.A, Javiad.S and Khalid.M.M, A new method to solve bi-objective transportation problem.International journal of applied science, 26(4)(2013):555- 563.

[6] Nomani.M.A, Ali.I, Ahmed.A, A new approach for solving multi-objective transportation problems. International Journal of Management Science and Engineering Management, 12(2017):165-173

[7] Haley.K, The solid transportation problem, operation research 10(1962):448-463.

[8] Pandian.P, KavithaAnuradha.D, A new approach for solving solid transportation problems, Applied mathematical Sciences 4 (2010):3603-3610.

[9] Pandian.P, Kavitha.K, Sensitivity analysis in solid transportation problems, Applied mathematical science, 6(136)(2012):6787-6796.

[10] Zadeh.L.A, Fuzzy sets. Inf. Control 8(1965): 338-353.

[11] Zimmermann.H.J, Fuzzy programming and linear programming with several objective functions, Fuzzy Sets system1 1(1978):45-55.

[12] Bit.A.K, Biswal.M.P and Alam.S.S, Fuzzy programming approach to multi-objective solid transportation problem. Fuzzy Sets Syst. 57(1993):183-194.

[13] Ojha.A, Das.B, Mondala.S and Maiti.M, An entropy based solid transportation problem for general fuzzy costs and time with fuzzy equality. Math. Comput. Model. 50(2009):166-178.

[14] Gupta.A, Kumar.A and Kaur.A, Mehar's method to find exact fuzzy optimal solution of unbalanced fully fuzzy multiobjective transportation problems. Optim. Lett. 6( 2012):1737-1751. 
[15] Deepika Rani and T.R.Gulati, Uncertain multi-objective multi-product solid transportation problems, Sadhana 41( 2016):531-539.

[16] Dipak Kumar Jana et al, Novel Multi objective multi item and four diemensional transportation problem with vehicle speed in LR type intuitionistic fuzzy environment,Neural computing and Applications,32(2020):11937-11955.

[17] B.Liu, Uncertainty Theory, 2nd ed., Springer-Verlag, Berlin, (2007).

[18] B.Liu, Uncertaint Theory: A Branch of Mathematics for Modeling Human Uncertainty, SpringerVerlag, Berlin, 2010.

[19] Yuan Gao, Uncertain models for single facility location problems on networks, Applied Mathematical Modelling 36(2012):2592-2599.

[20] B.Liu, Some Research Problems in Uncertainty Theory, Journal of Uncertain Systems, 3(2009):310.

[21] Bo Zhang, Jin Peng, Uncertain programming model for uncertain optimal assignment problem, Applied Mathematical Modelling 37(2013):6458-6468.

[22] B.Liu, Uncertainty theory, Springer, Berlin, Germany, 4th edition, (2013)

[23] X.Gao, Some Properties of Continuous Uncertain Measure,Internaltional Journal of Uncertainty,

Fuzziness and Knowledge-Based System,17(2009):419-426.

[24] SeyyedMojtabaGhasemi, Mohammad Reza Safi, Journal of Mathematical Analysis, 8(2)(2017):23-33.

[25] Liu, Uncertainty theory, Springer, Berlin, Germany, (2015).

[26] Liu.B, and Chen.XW Uncertain multi objective programming and uncertain goal programming, Journal of Uncertainty Analysis and Applications 3(1)(2015):1-8.

[27] Liu.B, and Yao.K Uncertain multilevel programming: Algorithm and applications, Computers and Industrial Engineering 89(2015):235-240.

[28] Zhou.J, Fan.Y, and Ke W Multi-objective optimization in uncertain random environments, Fuzzy Optimization and Decision Making 13(4)(2014):397-413.

[29] Zhong.S, Chen.Y, Zhou.J, and Liu.Y, An interactive satisficing approach for multi-objective optimization with uncertain parameters, Journal of Intelligent Manufacturing, 28(2017):535-547.

[30] Cui.Q, Sheng.Y, Uncertain programming model for solid transportation problem, An International InderdisciplinaryJournal,16(2(A))(2013):1207-1214.

[31] Guo et al, A Transportation Problem with Uncertain Costs and Random Supplies, International Journal of e-Navigation and Maritime Economy, 2(2015):1 - 11.

[32] yang et al, Reduction methods of type- 2 uncertain variables and their application to solid transportation problem, Information science, 291(2015):204-237.

[33] Hasan Dalman1, Uncertain Programming Model for Multi-item Solid Transportation Problem https://arxiv.org/abs/1606.00002, (2016).

[34] Yuan Gao and SamarjitKar, A solid transportation problem with product blending, International journal of fuzzy systems,19(2017):1916-1926.

[35] Y.Liu, M.Ha, Expected values of function of uncertain variables, Journal of Uncertain Systems,4(3)(2010):181-186.

[36] A.Charnes and W.W.Cooper, Goal Programming and Multiple objective, European Journal of Operation research,1(1977):39-54.

[37] T.Chang, "Muti-choicegoalprogramming",Omega, 35(2007):389-396.

[38] B.B.Pal, B.N.Moitra and U.Maulik, A goal programming procedure for fuzzy multiobjective linear fractional programming problem,Fuzzy Set Systems, 139(2003):395-405.

[39] R.H.Mohamed, The relationship between goal programming and fuzzy programming, Fuzzy Sets and Systems 89(1997):215-222.

[40] M.Zangiabadi and H.R.Maleki, Fuzzy goal programming technique to solve multi objective transportation problem linear membership function,Journal of Applied mathematics and Computing, 24(2007):449-460.

[41] M.Zangiabadi and H.R.Maleki, Fuzzy goal programming technique to solve multi objective transportation problem with some non-linear membership function,Iranian Journal of Fuzzy Systems, 1, (2013):61-74. 
[42] Bera S. GiriPK,Jana DK, Basu k, Maiti M, Multi-item D-TPs under budget constraint using rough interval, Appl Soft Computing 71 (2018),364-385. 